\section{Unusual cutaneous reaction with sodium valproate}

Sir

Cutaneous eruptions are one of the most common type of drug-related adverse reactions and accounted for $2-3 \%$ in hospital-based series. ${ }^{[1.2]}$ About $10 \%$ of patients receiving antiepileptic drug therapy develop skin allergy. ${ }^{[1]}$ Among the AEDs, sodium valproate is relatively free from skin allergy. This report describe a patient who developed sevee psoriasiform eruption with sodium valproate.

A 14 years boy was started on sodium valproate (extended release preparation) $500 \mathrm{mg}$ per day for recurrent left focal onset complex partial seizures following traumatic brain injury. Four months after starting sodium valproate he developed hair fall with white scaly scalp lesions. Gradually these lesions enlarged involving almost entire head and periauricular areas [Figure 1]. He also

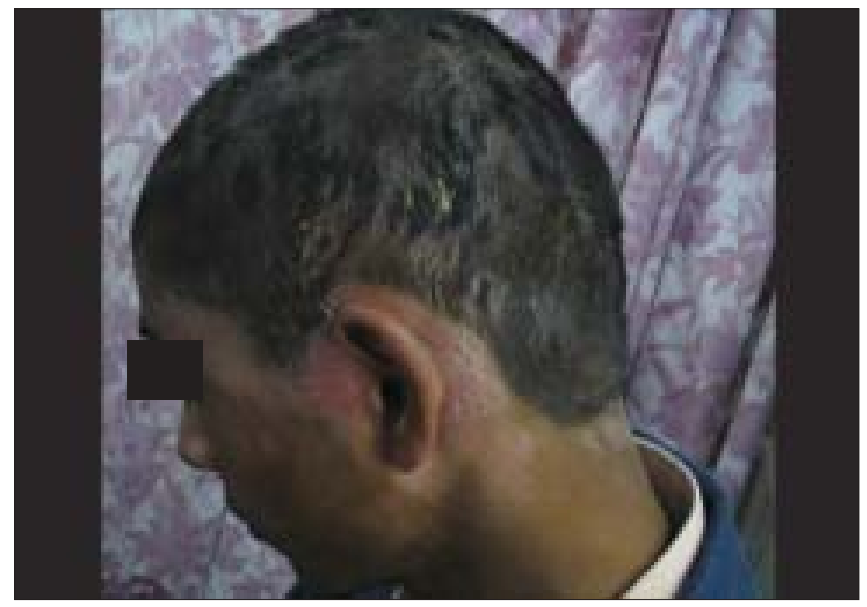

Figure 1: Scalp reaction with white scaly lesions

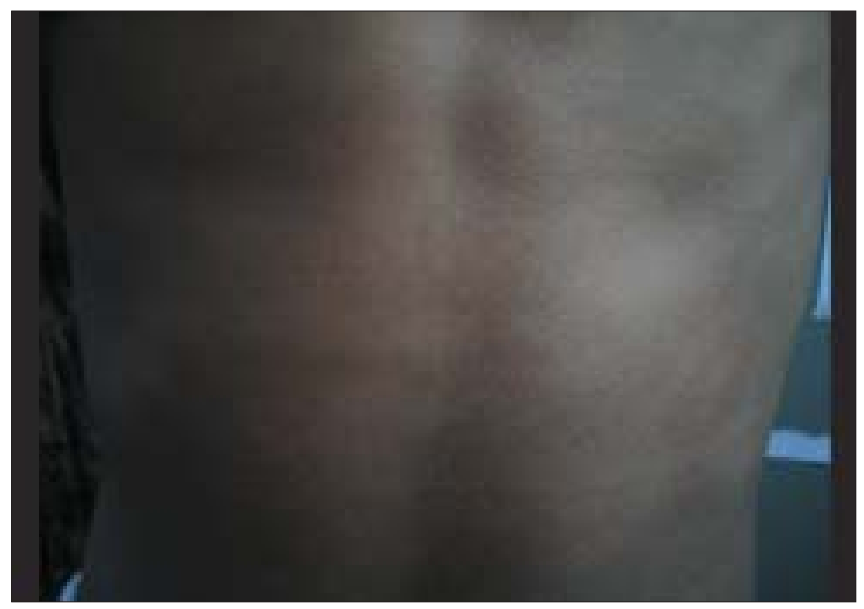

Figure 3: Recovery after drug cessation developed multiple discrete and confluent white scaly lesions with raised erythematous margins over the trunk, chest, and limbs [Figure 2]. The morphology of the lesions was suggestive of psoriasoform eruption.

Histology of the skin biopsy showed hyperkeratosis, parakeratosis, loss of granular layer, irregular acanthosis of the epidermis, and a perivascular infiltrate composed of mononuclear cells in the upper dermis. Patient was discontinued of valproate and was started on oxcarbazepine. The eruption completely disappeared in four months [Figures 3 and 4]. At 12 months follow up he was absolutely normal without any relapse of skin lesions.

Development of psoriasiform eruption with the initiation of valproate and subsequent remission of the lesions with the discontinuation of the drug and subsequent course clearly suggests a causal relation between valproate and skin lesions.

Various types of drug-related cutaneous eruptions

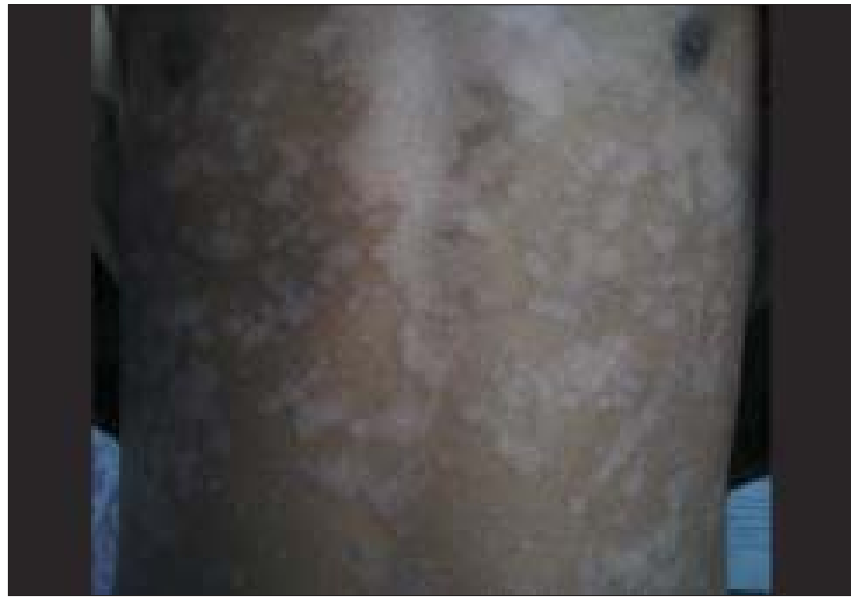

Figure 2: Abdominal wall with psoriasiform eruptions

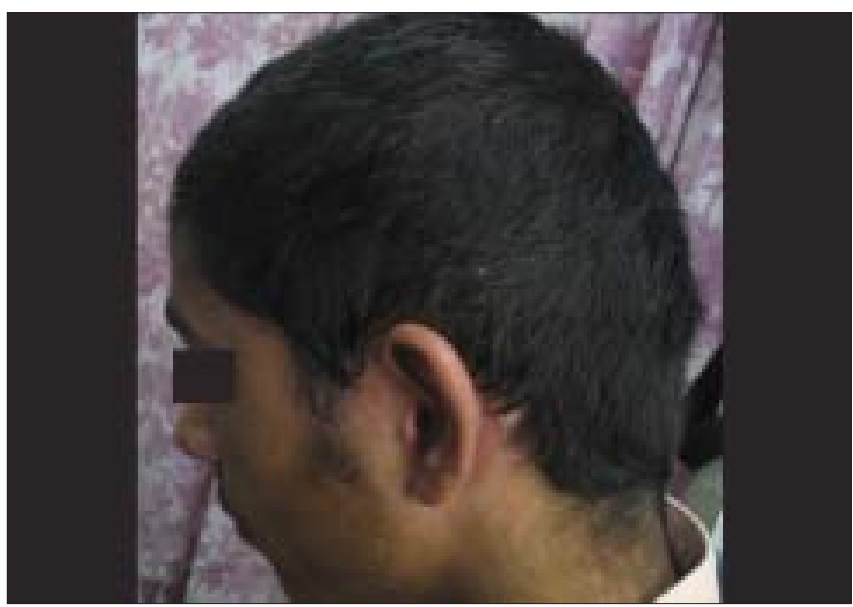

Figure 4: Recovery of scalp lesions 
include: Maculopapular rash, fixed drug eruption (FDE), erythema multiforme (EM), toxic epidermal necrolysis (TEN), Stevens-Johnson syndrome (SJS), urticaria, and erythroderma..$^{[1]}$ Maculopapular rash is the most common skin lesion with AEDs. The serious skin allergies include SJS and TEN. Skin allergies have been described more often in patients receiving carbamazepine, phenytoin and lamotrigine. ${ }^{[2]}$ Cutaneous eruptions are least with valproate compounds. Review of English literature showed only report of two cases of psoriasiform eruption with valproate. ${ }^{[3,4]}$

Drug treatment may result in exacerbation of pre-existing psoriasis, can induce psoriatic lesions on clinically uninvolved skin in patients with psoriasis, or can precipitate the disease in predisposed individuals. ${ }^{[5]}$ Our patient had no past or family history of psoriasis and no relapse of lesions at one year of follow up, thus suggesting that the psoriasiform eruption in him were probably related to valproate treatment. The knowledge of the drugs that may induce, trigger, or exacerbate psoriasis, is of importance in clinical practice. The drugs that may induce psoriasis include lithium, beta-adrenergic antagonists, antimalarial, non-steroidal anti-inflammatory drugs (NSAIDs) and rarely tetracycline. ${ }^{[6]}$ To this list we should add valproate and valproate should be used with caution in individuals with pre-existing psoriasis.

\section{S. Roy, Deepak Goel ${ }^{1}$}

Department of Dermatology and Neurology ${ }^{1}$, Himalayan Institute of Medical Sciences, Swami Ram Nagar, Dehradun (UT), India. E-mail:goeld007in@yahoo.co.in

DOI: $10.4103 / 0028-3886.51302$

\section{References}

1. Sharma VK, Dhar S. Clinical pattern of cutaneous drug eruption among children and adolescents in north India. Pediatr Dermatol 1995;12: 178-83.

2. Sharma VK, Vatve M, Sawhney IM, Kumar B. Clinical spectrum of drug rashes due to antiepileptics. J Assoc Physicians India 1998;46:595-7.

3. Brenner S, Wolf R, Landau M, Politi Y. Psoriasiform eruption induced by anticonvulsants. Isr J Med Sci 1994;30:283-6.

4. Brenner S, Golan H, Lerman Y. Psoriasiform eruption and anticonvulsant drugs. Acta Derm Venereol 2000;80:382.

5. Tsankov N, Angelova I, Kazandjieva J. Drug-induced psoriasis. Recognition and management. Am J Clin Dermatol 2000;1:159-65.

6. Dika E, Varotti C, Bardazzi F, Maibach HI. Drug-induced psoriasis: An evidence-based overview and the introduction of psoriatic drug eruption probability score. Cutan Ocul Toxicol 2006;25:1-11.

Accepted on 31-03-2009 